

\section{Editorial Introduction}

Our second edition of Health, Culture and Society, carries the title, Health System Dynamics and Barriers. This title was chosen to both bring to light the question of models, or modelization, at the same time as the obstacles which need to be understood and overcome by concerted efforts, to construct more integrational paradigms of access and delivery as well as culturally assertive strategies of health outreach.

Whether a country is building or reforming its health system, the undertaking is immensely challenging; the variables great. Policy strategy and legislation are historically sensitive, and the intensity of the trials and tribulations of an era will more often than not determine the material from which a health system can be crafted or rebuilt - this, reflected by research and its questions. Thus, today, analysis seeks to investigate determinants of a health system's performance with the goal of proposing policies and enhancing strategies that will improve performance and system consistency. Equity, access, delivery, are the key focus areas soliciting efforts; key structural and ethical challenges to the successful workability of a health system which involves, and indeed demands, a multilevel analysis of community, socio-economic and cultural variables. System strengthening and strategies are strong only when such variables are understood, and it is on the basis of this understanding that better outcomes can be had.

The collective enterprise of public health research is precisely where talents, experience and creatively acute energies engage with a landscape of complex and at times sensitive challenges; where there is the necessity to alleviate poverty, extend health access, and to increase if not guarantee quality of life and human rights. To alleviate, extend and guarantee are the ethical drives of our modern health research, particularly evidenced by the work of our contributors from Europe, Asia and the USA.

Progress towards the better performance of general health care delivery in rural areas as well as diseasespecific programs means that the question of social participation more than social inclusion is a key dimension to the processes defining the social value of a health care system, as is the role of our researchers, both in, and off, the field. This concerns the working realities and experiences of patients and professionals alike. Indeed, although system building and reform are reflected by a now widely employed terminology: affordability, equity, access, transparency, delivery - the research herein presented as the second issue of HCS articulates precisely what we really want the system to accomplish, precisely what is needed and precisely what we need to be thinking about. Contemporary urgencies, and the creative capacities and critical skills of those working and engaging with these, articulate how necessary more dynamic models of inclusion and accessibility are. Thus, it is important that HCS maintains and continues to extend its geographical coverage of issues affecting think-tank and policy strategy as well as continuing to grant visibility to the lived experiences of those in urban areas.

Policy makers strategize and design on the basis of performance, and performance can only be justly measured by both (1) the increase in wellbeing and, (2) the decrease of inequalities. Although these two factors to performance read very easily on paper, absorbing the great many experiences of peoples and professionals into the routine collecting of information and consequent analysis is a great challenge. This means that a definitive answer is not the goal, but rather the effectiveness in maintaining and improving quality of life not for the few, but for the many. This is the ethical imperative within a complex landscape of cultural, political, historical, social and economic interactions where policy and debate find meaning in the improvement of health and relief of suffering. This is the measure of performance, excellence and impact. 
We are, at the time of this writing, on the verge of the Sixty-fith World Health Assembly. We recall the Director-General's opening address almost one year ago at the Sixty-fourth World Health Assembly, urging Ministers and policy makers alike to "remember the people... in a world of enormous and constantly changing complexity." This was a clear, simple message with clear goals, defining an immensely challenging era. As Dr. Margaret Chan further made clear, gone are the days where thinking that hand-outs are the just and timely solution; this is no longer economically nor structurally viable. What countries sooner need are hand-ups in a world where food and fuel prices are spiralling out of control and where entire generations of the poor are increasingly at risk to disappearing altogether. Hand-outs are quickly dispensed at a distance - a look back at history will evidence this. Hand-ups require engagement, research, proximity and understanding. In this way, improving performance cannot be achieved without improving lives - this, the common mantra of our day.

We remember that not too long ago, on the back of the progressive transformation from centrally planned to market-oriented economies and reduced State intervention in national economies, there was another buzzword, namely, the "new universalism". This denoted new priorities, demands, values and challenges defining the global health system as well as facing governments on the eve of the new millennium. Now, in the wake of Dr. Margaret Chan's calls of 2011 (and they indeed resonate), resource-poor settings and the burdens of global diseases are not merely a question of finance, re-distribution and the soft rationing of services but of lives. Arguably, this brings to light the importance of prevention, which is sooner anticipation rather than precaution. Achieving better health for humanity requires an innovative model of prevention and greater incentives for solidarity within public health strategies. This is neither estranged from the new universalism nor from the insurers and State-providers, but a fundamental part of the scenario. The modelization of new public health models and strategies, can very much develop with initiatives of social and professional solidarity.

Thus, to retake, the importance of promoting visibility and maintaining and extending geographical coverage of research is a fundamental imperative of our journal, and to promoting a greater understanding of the issues affecting the global health system, as well as the barriers to be overcome.

Chapecó, Santa Catarina 